\title{
Using Quantum Dots to Simulate Magnetism
}

\author{
Researchers successfully use an array of quantum dots to create and study \\ a Heisenberg spin chain. \\ By Sophia Chen
}

$\prod$ he interaction of electron spins causes many interesting material properties, including magnetism and superconductivity. To better understand these properties, researchers would like to accurately simulate complex spin systems. That, however, is difficult to do on classical computers, as electron spin is a quantum-mechanical phenomenon, so researchers are instead turning to experimental "quantum simulators" to study these systems. Now, Cornelis Jacobus van Diepen and Tzu-Kan Hsiao of Delft University of Technology in the Netherlands and colleagues have experimentally simulated a simple magnetic system using a platform based on quantum dots [1]. While the platform is small enough to numerically simulate on a classical computer, the researchers say their demonstration indicates the viability of quantum dots to probe large-scale spin systems.

The team's quantum simulator consists of a sandwich of two semiconductors, gallium arsenide and aluminum gallium arsenide. At the interface between the two materials, there are electric potentials that mimic the positive charge of an atomic

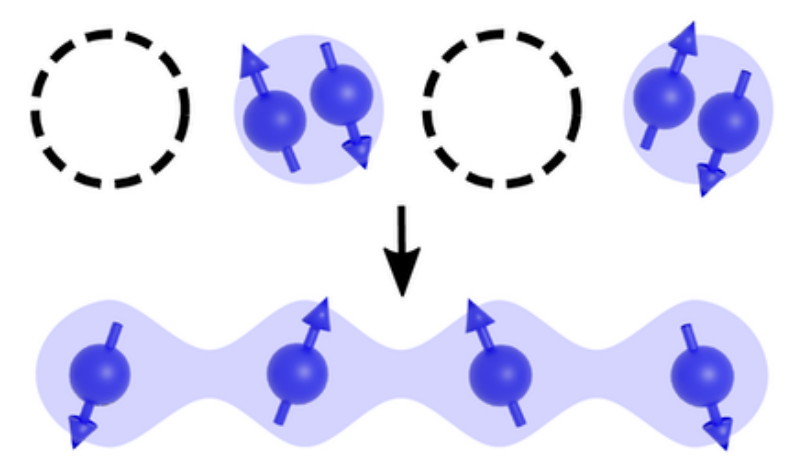

nucleus. These artificial atomic "nuclei” trap electrons, creating quantum dots.

By tuning the interactions between the electrons in four neighboring quantum dots, the team simulated the behavior of a four-electron "Heisenberg spin chain," with each quantum dot holding one electron spin. They characterized the system by initializing it in a certain state. Then, they measured the energy of the spins, finding they matched predictions from classical numerical simulations. In addition, they made the spins in the spin chain oscillate in a manner predicted by theory.

In future work, the team says that they plan to simulate spin systems arranged in different lattice configurations. They also hope to investigate using other semiconductors to create their platform, with the aim of making a magnetically quieter environment for the quantum dot spins.

Sophia Chen is a freelance science writer based in Columbus, Ohio.

\section{REFERENCES}

1. C. J. van Diepen et al., "Quantum simulation of antiferromagnetic Heisenberg chain with gate-defined quantum dots," Phys. Rev. X 11, 041025 (2021).

Credit: C. J. van Diepen et al. [1] 